\title{
An electron diffractometer: A novel device for electron crystallography experiments
}

\author{
G. Steinfeld, A. E. Lanza, E. Hovestreydt, G. Santiso-Quinones \\ ELDICO Scientific AG, 5234 Villigen, Switzerland \\ steinfeld@eldico.ch
}

Electron diffraction experiments and electron crystallography are experiencing a major leap in the nanocrystallography revolution [1]. Moreover, the use of the continuous rotation method (as in X-ray crystallography) for the collection of electron diffraction data is surpassing all previous data collection methods available in the past decade [2].

Unfortunately, there is no dedicated device for performing such experiments. All experiments found in the literature are done in (modified)-Transmission Electron Microscopes, as these are the only sources of electron beams. Though these devices are not optimal for performing such kind of experiments. Many factors play an important role here. In fact, scientist interested in using this technique, have even suggested on guidelines to use a (S)TEM as an electron diffractometer [3].

Therefore, there is a huge necessity that fully integrated electron diffractometers are available for the scientific community and industrial facilities. This necessity is now a reality. Here we present a novel device optimized and dedicated for electron diffraction experiments that uses the continuous rotation method. We will showcase this device and its advantages against electron microscopes that can perform ED measurements. We will show experimental evidence on the improvement of the data quality compared to data sets collected elsewhere. We will highlight the ease of use of this device.

[1] Gemmi, M., Mugnaioli, E., Gorelik, T. E., Kolb, U., Palatinus, L., Boullay, P., Hövmoller, S. and Abrahams, J. P. (2019). ACS Cent Sci., 5, 1315-1329.

[2] Source: Paul Klar, Institute of Physics of the CAS, Prague, April 2021.

[3] Heidler, J., Pantelic, R., Wennmacher, J. T. C., Zaubitzer, C., Fecteau-Lefebvre, A., Goldie, K. N., Müller, E., Holstein, J. J., van Genderen, E., De Carlo, S. \& Gruene, T. (2019). Acta Cryst. D75, 458-466.

Keywords: electron diffractometer; electron diffraction; electron crystallography; nanocrystallography 